\title{
The performance of a wire mesh collimator SPECT camera for different breast volumes in prone position
}

\begin{abstract}
The multihole collimator is the most commonly used collimator in conventional SPECT cameras for general purpose imaging. However, there are some limitations with this collimator, which includes the lack of sensitivity as a trade-off for obtaining better spatial resolution. This paper looks at the performance of a wire mesh collimator that was introduced recently in order to improve the ability of SPECT cameras in mapping breast cancer cells, utilizing the Technetium-99 m $140 \mathrm{keV}$ radiotracer. In this work, various volumes of breast are modelled and simulated using Monte Carlo N-Particle (MCNP5) code, derived based on the real cup sizes and volumes in prone position. The size of tumour is $1 \mathrm{~cm}$ in diameter with tumour to background ratios (TBRs) ranging between TBR from 1:1 to TBR 20:1, and located $2 \mathrm{~cm}$ inside breast skin. The results show that wire mesh collimator 1 (WM-1) has the highest sensitivity and signal to noise ratio (SNR) in comparison with wire mesh collimator 2 (WM-2) and the multihole collimator (MHC). This indicates the potential of using a wire mesh collimator for early mapping of breast cancer cells.
\end{abstract}

Keyword: Wire-mesh collimator, Multihole collimator, SPECT camera, TBR, SNR, Breast, Prone position 